\section{Aporte de la etnografía en el conocimiento de los códigos socioculturales de la leishmaniasis cutánea localizada en un programa de educación para la salud, en Venezuela}

\author{
The contribution of ethnography to knowledge \\ on socio-cultural codes related to localized \\ cutaneous leishmaniasis in a health \\ education program in Venezuela
}

Baílde García Guevara 1

\section{Introducción}

This article presents the experience with the validation of the ethnographic methodology as an alternative for an objective and timely approach to the construction of socio-cultural scenarios in communities affected by cutaneous leishmaniasis in Venezuela. It begins with a brief presentation on the resurgence of leishmaniasis in the Americas as a whole and in Venezuela in the context of social inequalities. It proceeds with a review of the role of health education in programs to control endemic diseases. It then highlights the role of ethnography in the construction of rationality in daily life, its meanings, and interpretation by persons with the disease in order to elaborate permanent and sustainable health education programs. The methodology was validated using a figure in which an analysis was performed of the clinical and epidemiological history normally used to gather information on the disease. Among the main findings was the inadequacy of the clinical history design for obtaining information on socio-cultural factors linked to the disease, expressed by specific geographic territories with the support of georeferencing.

Health Education; Consumer Participation; Cultural Anthropology; Leishmaniasis; Social Inequity
De acuerdo con la Organización Panamericana de la Salud (OPS) en su publicación correspondiente al cuaderno número 44 dedicado a la epidemiología y control de la leishmaniasis en las Américas por país o territorio (año 1996), la leishmaniasis es un problema de salud pública que durante los últimos 10 años plantea que el número de personas afectadas por las distintas formas clínicas de la leishmaniasis en el continente americano ha aumentado notablemente, sobre todo en América Central y en algunas zonas de América del Sur 1 .

Esta situación presentada parece despertar en los últimos tiempos una sensibilidad más aguda en las discusiones científicas en la región, constituyendo así un tema obligado en diversos foros nacionales e internacionales (Organización Mundial de la Salud/OPS). La tendencia en cuanto a los resultados de estos encuentros se ubica en apreciar que el resurgimiento de las diferentes endemias, y particularmente la leishmaniasis, está asociado a un comportamiento multifactorial: clínico-entomológico-epidemiológico, así como a la profundización de las inequidades y desigualdades sociales, aspectos considerados básicos para el desarrollo humano y la justicia social. Se expresa de diferentes formas, entre ellas: (1) la igualdad de oportunidades de los individuos y comunidades en tener acceso y utilización oportuna a los servicios de salud de acuerdo a sus 
necesidades; (2) ausencia de políticas sostenibles en el control integral de las endemias; (3) poca capacidad de coordinación transectorial; (4) insuficientes investigaciones operacionales en epidemiología, educación y participación comunitaria; (5) déficit o ausencia de un sistema básico de servicios públicos; y (6) el deterioro progresivo del medio ambiente, entre las más importantes.

De igual manera, juega un papel importante lo relacionado con formas de vida de las poblaciones; aperturas indiscriminadas de complejos turísticos, industriales y comerciales en áreas endémicas, sin considerar las medidas de prevención; aumento acelerado del desempleo, ocasionando en muchos casos migraciones de personas a centros poblados con presencia de focos activos de leishmaniasis, y en la mayoría los casos sin ningún tipo de información de cómo protegerse ante la endemia.

El análisis de estos factores causales en el resurgimiento de las enfermedades infecciosas en las Américas necesariamente debe verse a la luz del contexto del papel del estado y de las diferentes reformas sociales, políticas, económicas y sanitarias por las cuales la mayoría de los países de la región están cruzando.

Venezuela, como país de la región de las Américas, igualmente se encuentra amenazado por cada uno de los factores anteriormente mencionados. Al analizar los datos nacionales de enfermos de leishmaniasis (año 2002), reportados por el Servicio Autónomo Instituto de Biomedicina, organismo dependiente del Ministerio de Salud y Desarrollo Social y de la Universidad Central, responsable de la gestión de los programas de control y prevención de la mencionada enfermedad en el país, se observa que la presencia de la misma se encuentra en casi todas las Entidades Federales, con las únicas excepciones del Estado Nueva Esparta y el Estado Delta Amacuro 2.

Conforme estos datos, se ha hecho una clasificación inicial de las entidades federales del país: Anzoátegui, Cojedes, Lara, Mérida, Miranda, Sucre, Táchira, Trujillo y Yaracuy. Son focos muy activos: Aragua Barinas, Bolívar, Carabobo, Distrito Federal, Falcón; son focos activos: Monagas, Portuguesa, y Zulia; Apure, Amazonas y Guárico son focos discretamente activos.

La leishmaniasis en Venezuela presenta sus tasas más elevadas en las zonas correspondientes al pie de monte de los sistemas orográficos, donde se dan las condiciones ecológicas para la persistencia de la cadena epidemiológica de la enfermedad.

El programa de control de la leishmaniasis desde la década de los cuarenta siempre había sido realizado con registros manuales, generando algunas dificultades en cuanto a la obtención de datos pertinentes y confiables para la planificación y toma de decisiones. En consecuencia, a partir de la década de los 80 , se inicia el proceso de automatización de los datos clínicos y epidemiológicos de todo el país, abriéndose el camino de un registro más objetivo, oportuno y confiable para la gestión del programa. Este cambio es fortalecido por la instalación de los sistemas de georreferencia como herramienta para la organización en forma espacial-social y estratificada del comportamiento de la leishmaniasis por municipios y parroquias de todo el país. Este aporte tecnológico incorpora en el análisis clínico y epidemiológico la categoría de la espacialidad desde una perspectiva socio-histórica de la problemática. La misma contribuye a la caracterización y comportamiento de las diferentes variables que condicionan la presencia y distribución de la enfermedad, a través de la incorporación de género y ocupación, grado de instrucción, edad, niveles de procedencia, formas de vida, modos de producción económica y social.

El concepto de espacialidad también es planteado desde la epidemiología crítica social por parte de Breilh 3 , cuando expresa que la geografía deviene frente a la salud, no en un simple reservorio de climas contaminantes con microbios, de vectores de transmisión infecciosa, etc., sino en un espacio históricamente estructurado, donde también se expresan las consecuencias benéficas y destructivas de la organización social.

Estas premisas contextuales de la epidemiología contemporánea relativa al proceso saludenfermedad llevan a una revisión del patrón clásico de la epidemiología de la leishmaniasis, donde el énfasis está en el análisis de los factores de riesgos individuales, sin profundizar sobre las profundas transformaciones socioeconómicas de las sociedades. Referencia que permite pensar en la perspectiva de una epidemiología históricasocial, que facilite el análisis de la presencia de la endemia en las comunidades, desde la integración de la multiplicidad de factores estructurales que generan los posibles riesgos en grupos de población que anteriormente no eran considerados, y los cuales están asociados a factores vinculantes a las formas de vida, producción económica y sociocultural, enmarcados en los cambios políticos de la sociedad global. 


\section{Breve revisión histórica del papel de la educación para la salud en los programas de control de endemias en Venezuela}

La educación para la salud y participación comunitaria han estado vinculadas a las políticas y programas de salud pública, así como para el control de las diferentes endemias en Venezuela desde el mismo momento de la creación del Ministerio de Sanidad y Asistencia Social (año 1936), teniendo su génesis en una visión filosófica y conceptual desde el paradigma de Educación Sanitaria, siendo su naturaleza asistencialista y benéfica, vinculante a la interpretación de la salud como biologicista-unicausal, sólo como ausencia de enfermedades. Esta interpretación históricamente ha venido cambiando, al menos teóricamente, a partir de la década de los 60 por las transformaciones sociales y políticas del país, momento a partir del cual se inicia la discusión y apertura hacia el paradigma de la educación para la salud, que rompe estructuralmente la esencia filosófica y metodológica del paradigma anterior. Interpretándose, entonces, como una de las estrategias básicas en el marco de la calidad de vida y promoción de la salud en el desarrollo social sostenible del país.

Igualmente, en el ámbito internacional suceden en esta década eventos importantes que dan reconocimiento y fortalecimiento a la educación para la salud en las políticas públicas de salud en los países que conforman la región de las Américas; contribuyendo de esa manera a abrir un espacio de discusión y de propuestas relacionado con esta temática en el marco de las políticas de salud en Venezuela.

De estos encuentros se citan la Declaración de la Atención Primaria en Salud en Alma-Ata, Rusia (1978), seguido por la Carta de Ottawa, Primera Conferencia Internacional de Promoción de la Salud, en Ottawa, Canadá (1986). A esta Conferencia le siguieron otras que examinaron los temas más destacados tratados en la Carta de Ottawa sobre una política pública saludable (1988), Cuarta Conferencia Internacional de Promoción para la Salud, Yakarta, Indonesia (Julio 1997), así como otros encuentros. En cada uno de estos encuentros la variable constante ha sido la educación y la participación comunitaria como estrategia esencial para contribuir en la transformación de salud de las comunidades.

Estas referencias marcan de igual manera el comportamiento de la educación para la salud en el campo de los programas de control de endemias en el país, que ha sido interpretada y aplicada desde la visión de educación sanitaria, orientando los esfuerzos técnicos y humanos desde una postura centralizada de la planificación, con sentido unidireccional-coyuntural-benéfico para la consecución de objetivos específicos en cuanto al control de ciertas enfermedades, con especial énfasis en momentos de crisis o repunte de las mismas.

En la década de los 40 y 50 se crean los Servicios de Dermatología Sanitaria a nivel nacional, y con ello la instalación del registro manual del Programa de Leishmaniasis, marcado por el centralismo y la verticalidad de las acciones clínicas. Progresivamente, se van conformando equipos de trabajo en cada una de estos servicios estrictamente biomédicos, los cuales reciben directrices para ejecutar actividades de educación sanitaria enmarcadas en la prevención, promoción y fomento de la salud inscritas en el modelo de Leavell \& Clark ${ }^{4}$, el cual explica la salud como la evolución natural de la enfermedad y la expresa en tres niveles de atención médica, en correspondencia con el grado de complejidad de la patología. Vinculado a una visión y praxis de una educación bancaria, donde la población (pacientes) sólo constituyen sujetos pasivos de la información sanitaria y de las acciones médicas. Ahora bien, este modelo obtuvo algunos resultados sanitarios coyunturales en cuanto a la reducción de estas endemias en aquel momento, pero sin capacidad resolutiva permanente y sostenible en la colectividad. En consecuencia, como plantea Freire 5 , el acto educativo no puede quedar reducido al puro acto informativo o de instrucción, no puede decirse educativo lo que tiene un sentido unidireccional. Es el conjunto de interacciones, que se generan en el proceso vital de relación, el que llena toda la riqueza formativa; es la relación dialógica y crítica el sello del proceso gnoseológico.

A partir de la década de los 90 es cuando se crea la Unidad de Educación para la Salud y Participación Comunitaria en el marco del control de las endemias en el Instituto de Biomedicina, marcando de esa manera la integración de las ciencias sociales en las dimensiones de la gestión de los programas de control de la leishmaniasis a nivel nacional.

Desde ese momento se han realizado proyectos de educación para la salud en sus diferentes dimensiones: investigaciones operacionales, específicamente en el marco del protocolo de inmunoprofilaxis de leishmaniasis en el Municipio Andrés Eloy Blanco, año 1992. Diseño y ejecución de formación de personal de salud (promotores sociales), actualización del personal de salud en servicio en metodologías de educación para la salud y participación comunitaria, y otras acciones que han permitido ir abriendo un espacio de discusión y reflexión del papel de la educación y 
participación comunitaria en las comunidades endémicas de la leishmaniasis.

\section{La etnografía y su contribución a los programas de educación para la salud en leishmaniasis cutánea localizada}

Por la naturaleza del tema en referencia se ha seleccionado una metodología cualitativa. La etnografía representa una alternativa para aproximarnos de manera objetiva y oportuna a la construcción de los servicios de salud y de las comunidades desde los propios escenarios socioculturales, así como a las iniciativas de educación para la salud y participación comunitaria.

La etnografía, como análisis de la vida cotidiana, necesariamente abarca lo cultural, ya que ésta se crea y recrea en los espacios de encuentro en sus dimensiones y espacios culturales y lingüísticos; entre éstos la praxis de los profesionales de la salud, como espacio de encuentro intercultural cotidiano 6 .

Las características de este método permiten su relación con el modelo de la epidemiología crítica contemporánea, la cual plantea el proceso salud-enfermedad en una dimensión sociopolítica e histórica de las sociedades, así como con la georreferencia tecnología, que facilita la caracterización de las poblaciones de forma espacial-estratificada, permitiendo la identificación y relación de los diferentes indicadores de salud, socio-ambientales, culturales, institucionales, políticos, económicos, y niveles de organización social comunitaria. Se puede realizar a través de procesos manuales (sectorización), que arrojan mapas básicos, y a través de procesos automatizados (sistemas de información geográfica), los cuales derivan en mapas temáticos por municipio, parroquia, comunidades, sectores, sub-sectores. Todo ello es base para representar los códigos culturales asociados a la leishmaniasis.

\section{Metodología}

La aplicabilidad del método etnográfico facilita la construcción de la georreferencia de los factores socioculturales, asociados al comportamiento de la leishmaniasis en los estados endémicos en Venezuela, una manera objetiva de generar iniciativas de educación y participación comunitaria de forma permanente y sostenible.

En este caso es planteada desde la estrategia de taller de discusión-reflexión con el personal de salud ubicado en los servicios de dermatología sanitaria del país. La elección de este personal, como unidad de análisis de la investigación, obedece a un perfil de conocimiento de la endemia y por su amplísima experiencia derivada de un largo periodo de trabajo en el programa de control de la leishmaniasis.

A efectos de la presentación de este artículo nos referiremos a los resultados preliminares obtenidos durante el taller realizado con un universo de población de (30) profesionales de diversas disciplinas de las ciencias de la salud: médicos dermatólogos, inspectores de salud, promotores de salud, enfermería, trabajadoras sociales, procedentes de 7 estados de la región centro-occidental del país: Mérida, Trujillo, Táchira, Lara, Falcón, Zulia y Yaracuy.

\section{Recolección de información}

Los instrumentos seleccionados para la recolección de la información durante el desarrollo del taller, posiblemente no sean los convencionales en un proceso de investigación, se refieren a la historia clínica y epidemiológica de la leishmaniasis, registro obligatorio y generalizado en los diferentes servicios de dermatología sanitaria en Venezuela, así como a la georreferencia aplicada en las comunidades de influencia endémicas de la leishmaniasis.

Los criterios de selección de estos instrumentos obedecen a que son registros básicos y permanentes en los programas de control de la leishmaniasis; y a su vez, son vinculantes con los objetivos de la etnografía aplicada en el campo de la salud pública, donde la historia clínica es considerada como un registro importante, cuando en ella quedan plasmadas los signos con sus significados de las dimensiones de la enfermedad en un contexto cultural del paciente, entendido éste como familiar y comunitario, al igual que la importancia de la caracterización espacial-estratificada de las poblaciones involucradas en el proceso investigativo.

Para operacionalizar los mencionados instrumentos durante el taller se diseñó una guía de preguntas abiertas relacionadas con: icuáles son los objetivos y alcances de la historia clínica y epidemiológica en su servicio?, ¿se realiza la georreferencia en su servicio, y ¿cuál es la utilidad?, ¿qué acciones de educación para la salud se realizan en los diferentes servicios?, y ¿cuál es su orientación en el diseño, ejecución y evaluación? 


\section{Resultados}

En cuanto a la primera pregunta: ¿cuáles son los objetivos y alcances de la historia clínica y epidemiológica en su servicio? La tendencia de los resultados arroja que sus objetivos están dirigidos a la recolección de información clínica y epidemiológica de la enfermedad, con una visión estrictamente biomédica hacia la consecución de la construcción de un registro institucional para el diagnóstico clínico, aplicación y evaluación del tratamiento, de acuerdo con la magnitud del daño en cada uno de los pacientes. El diseño del mismo no permite la recolección de información relativa a los factores socioculturales vinculantes a la enfermedad. A este respecto, la totalidad de los participantes manifiestan que los pacientes, básicamente en la primera consulta, expresan a veces de manera explícita, o en otros casos de forma muy tímida la significación cultural de la enfermedad asociada a sus angustias, tristezas, molestias y temores, entre otros aspectos psico-afectivos. Ahora bien, según los mismos participantes, esta información no tiene ninguna relevancia como insumo formal para la apertura y seguimiento de la historia clínica, sólo representa un hecho marginal, que es solamente considerada en aquellos casos, cuando existe por parte de los profesionales de la salud una actitud positiva al respecto. En consecuencia, queda explícita la materialización de las limitaciones del diseño actual de la historia clínica y epidemiológica para efectos del registro de los factores socioculturales de la mencionada endemia, contándose solamente con testimonios profesionales de la salud que guardan un registro oral muy valioso, pero sin ningún tipo de aplicación en el diagnóstico, tratamiento y evaluación del proceso y resultados de la enfermedad en el paciente.

Las debilidades que presenta la actual historia clínica y epidemiológica en el diseño de programas de educación para la salud y participación comunitaria para el control integral de leishmaniasis pueden convertirse en una fortaleza, si se consideran como un recurso institucional de referencia a la hora de abrir un proceso de discusión y de redefinición de la misma con el objetivo del diseño de una historia integral. A partir de esto se pueden articular las variables clínicas-epidemiológicos-socioculturales, éstas últimas desagregadas en cuanto a su asociación directa al mundo psico-afectivo de cada paciente, al impacto en la aplicación y cumplimiento del tratamiento, y por consiguiente, en el proceso y resultados de la curación. Además, permite identificar los contextos de los hábitos, costumbres y creencias que las diferentes personas pro- ducen y reproducen en la vida cotidiana, esencia de la etnografía.

De lo que se trata entonces, es que a través de una interacción respetuosa y ética se abra un canal de reflexión-discusión con los equipos locales de salud para lograr un proceso de cambio hacia la comprensión clínica y epidemiológica de la leishmaniasis en una dimensión crítica histórica y social, y de esa manera, poder integrar la perspectiva de educación para la salud y participación comunitaria, de acuerdo a un análisis objetivo de los diferentes tipos de poblaciones en correspondencia a criterios de espacialidad geográfica-epidemiológica y social, obteniéndose una lectura de necesidades sociales configuradas por territorios específicos.

Por lo tanto, cuando se plantea un diseño y aplicación de una historia integral del paciente de leishmaniasis con las características mencionadas, el objetivo es lograr la posibilidad concreta de la aplicación del método etnográfico como vía para la construcción de un sistema de georreferencia sociocultural, relacionado con la leishmaniasis desde los servicios de consulta y desde las comunidades endémicas.

En la Figura 1 se presenta, a manera de ejemplo, una gráfica de la historia integral, y los aportes conceptuales y metodológicos que de ella se derivan.

Los resultados de la aplicación de cada historia integral del paciente en los diferentes servicios de dermatología sanitaria deben retroalimentarse de forma permanente con las diferentes acciones de educación para la salud y de vigilancia epidemiológica en las comunidades de influencia de los respectivos servicios. En este caso, la georreferencia (bien sea manual o automatizada) puede constituir uno de los elementos claves de esa relación (Figura 1).

Al considerar el contenido de la segunda pregunta $i$ se realiza la georreferencia en su servicio?, y ¿cuál es la utilidad? El grupo en su conjunto expresa no tener conocimiento, ni experiencia en la georreferencia moderna basada en los sistemas automatizados y que solamente conocen y manejan en los servicios de dermatología sanitaria el modelo de la georreferencia básica-manual y de manera empírica. La misma es expresada en mapas a mano alzada, donde se representa en forma de gráficas la caracterización general de la población endémica de influencia a los servicios y, particularmente, la identificación general de los diferentes focos activos de la leishmaniasis en el estado o municipio en referencia. De igual manera, programar, monitorear y evaluar el tratamiento de los enfermos.

No obstante, estas experiencias del grupo (registros orales) son muy valiosas para aproximar- 
Figura 1

Propuesta de educación para la salud y participación comunitaria en leishmaniasis, aspectos conceptuales metodológicos.

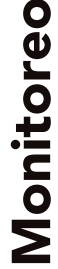

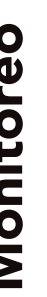

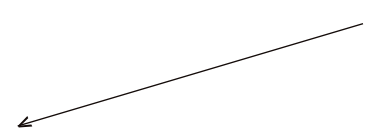

Escenario 1, individual

Reformulación de las historias

clínicas/relatos de vida

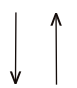

Población (pacientes)

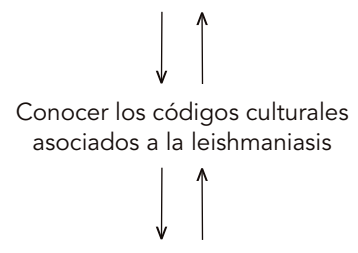

Análisis de la significación psico-afectiva de cada código cultural

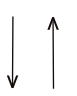

Impacto de la significación psico-afectiva en el comportamiento del tratamiento y posibilidades de curación

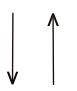

Construcción del mapa temático, representaciones culturales y su significación psico-afectiva

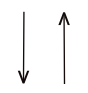

Generación de un registro informatizado para iniciativas de educación para la rehabilitación, cumplimiento del tratamiento y apoyo psicológico
Método etnográfico

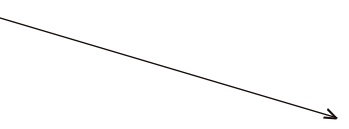

Escenario 2, colectivo

Conformación de grupos focales en comunidades endémicas de leishmaniasis
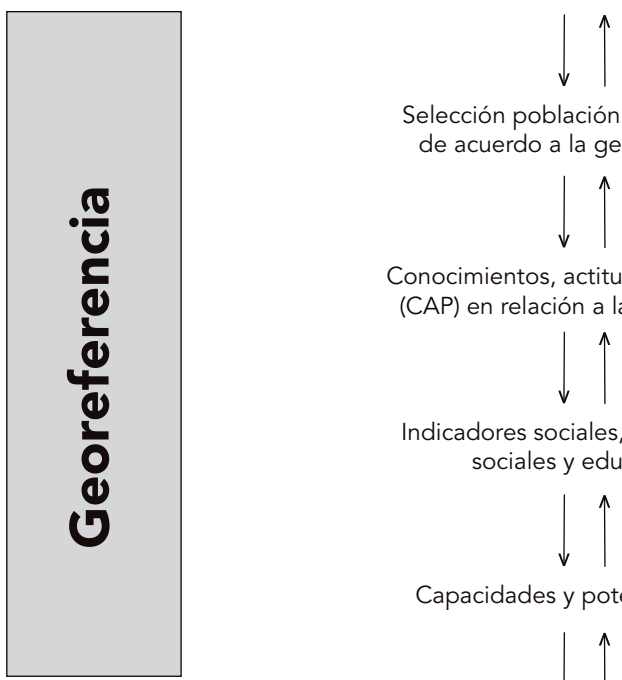

Selección población por sectores, de acuerdo a la georreferencia

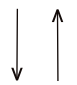

Conocimientos, actitudes y prácticas (CAP) en relación a la enfermedad

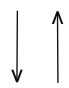

Indicadores sociales, económicos, sociales y educativos

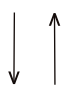

Capacidades y potencialidades

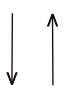

Análisis del perfil integral comunitario

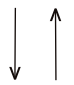

Construcción del mapa temático epidemiológico y social por sector en las diferentes parroquias

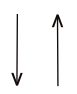

Generación de un registro para la organización de grupos de vigilancia epidemiológica y de educación para la salud en cada uno de los sectores de las diferentes parroquias

Retroalimentación

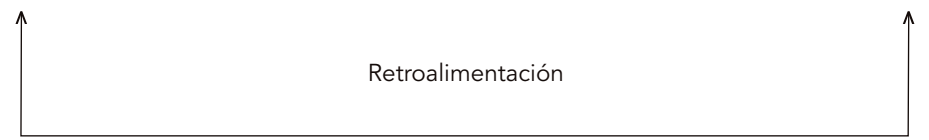

Sostenibilidad 
nos a la comprensión de un análisis espacial del comportamiento de los factores socioculturales asociados a la leishmaniasis por estado.

A partir del relato de los participantes se elabora un mapa de representaciones socioculturales asociado a la leishmaniasis (Figura 2) donde se pueden observar las diferentes expresiones culturales asociadas a la enfermedad y sus diferencias entre estados, y además con una diversidad de vinculaciones psico-afectivas. Estas vinculaciones aunque no aparezcan en forma gráfica en el mapa, los informantes las generan según sus experiencias cotidianas, un impacto favorable o no favorable en la curación del paciente. A efectos demostrativos de estas premisas, se presentan algunos ejemplos utilizando para ello referencias de 3 estados.

1) Categoría sociocultural de la leishmaniasis llaga. Asociada al mundo psico-afectivo de los pacientes de diversas maneras, de acuerdo con el lugar donde residen: vergüenza, tristeza, dolor, irrelevante. Su impacto favorable o no con el tratamiento y la curación se relaciona en gran medida al tipo de las categorías descritas.

En el caso de los pacientes que la definen como algo irrelevante, los informantes (médicos dermatólogos) manifiestan que este grupo refleja una tendencia hacia un escenario de tranquilidad, casi de indiferencia ante la enfermedad, sin demostración aparente de angustia o temor. Este tipo de grupo de acuerdo a la experiencia de los participantes se divide en dos categorías de pacientes: un grupo que no asume al inicio del diagnóstico clínico seriedad y responsabilidad para el cumplimiento del protocolo de tratamiento, aspecto que tiende a ser superado una vez que el profesional de la salud le explica el proceso de la enfermedad y la importancia de la aplicación de los medicamentos indicados para el proceso de recuperación de la salud; y otro grupo con una actitud más positiva, asumiendo con tranquilidad pero con responsabilidad el diagnóstico clínico y tratamiento asignado por el médico. En este tipo de grupo resalta la confianza y la tranquilidad de la recuperación de la salud. Los resultados en general se ubican en escala favorable en tiempo de recuperación de acuerdo a parámetros clínicos esperados.

El grupo que identifica la leishmaniasis con las categorías de rechazo, vergüenza, el escenario psicológico es otro. Se sienten deprimidos con mucha ansiedad-tensión y hasta desconfianza en el diagnóstico clínico y el tratamiento, dando como resultado un proceso más lento en la curación. La experiencia del grupo indica que en este tipo de pacientes la comunicación amena y permanente da muy buenos resultados para
Figura 2

Mapa de representaciones socioculturales asociado a la leishmaniasis.

Códigos culturales $\Longleftrightarrow$ Aspectos psico-afectivos del paciente $\Longleftrightarrow$ Impacto tratamiento y curación

minimizar el grado de tensión y ansiedad presentado al inicio de la primera consulta.

2) Categoría sociocultural de la leishmaniasis $l e$ pra. Está asociada a la muerte bíblica, al rechazo social, vergüenza. Según los informantes - médicos - estos pacientes desde la primera consulta confiesan que están muy mal que pueden hasta morir, porque ellos saben que tienen una enfermedad muy grave (que es la lepra). El proceso tanto de diagnóstico, como de aplicación y cumplimiento del tratamiento, es algo difícil, pues se hace necesario convencerles de que su enfermedad no es lo que ellos piensan, y que no van a morir. En consecuencia, en estos casos los resultados de la curación por lo general son muy lentos, e incluso abandonan el tratamiento, lo que implica situarlo en sus hogares para motivarles en la continuidad del tratamiento.

3) Categoría sociocultural de la leishmaniasis llaga comilona. Ésta se asocia a factores mágicos y religiosos. El perfil de pacientes correspondientes a este grupo presenta una tipología donde se enfatizan aspectos de daños o brujerías que alguien, por alguna razón, les ha hecho, y que, por lo tanto, requieren la asistencia de una persona yerbatera que les ayude a curarse. La tendencia en este grupo de personas es buscar atención médica cuando ha pasado algún tiempo y no mejoran con los tratamientos caseros, y la lesión o lesiones se encuentran, por lo general, en un estado avanzado. En algunos casos durante el proceso de tratamiento logran entender cuáles son las causas clínicas que dieron origen a la enfermedad, pero en otros casos persiste la idea que su enfermedad es producto de un daño o brujería.

Esta visión preliminar de una georreferencia de factores socioculturales, asociados a la leishmaniasis por estados y municipios, a partir de información empírica brindada por los participantes en el taller (en su mayoría médicos dermatólogos), a partir de datos manejados desde la cotidianidad de sus servicios, constituye una motivación para iniciar un proceso de análisis espacial-estratificado, tanto geográfico-epidemiológico y social de la enfermedad, a partir de la utilización del método etnográfico. En correspondencia a estas premisas se puede considerar 
un escenario favorable para diseñar iniciativas de educación para la salud y participación comunitaria de manera más objetiva a los diferentes públicos/objetivos, tanto de pacientes como de la población en riesgo de enfermar.

$\mathrm{Al}$ ser contrastadas estas consideraciones con el contenido de la tercera pregunta: ¿qué acciones de educación para la salud se realizan en los diferentes servicios?, y ¿ cuál es su orientación en el diseño, ejecución y evaluación? Se conduce al grupo a una revisión del modelo conceptual y metodológico que sustenta las acciones de educación que regularmente se desarrollan en el marco del programa de control de la leishmaniasis desde los diferentes servicios, como en las comunidades de influencia de los mismos.

El análisis del grupo se orienta hacia el paradigma de educación sanitaria enmarcado en los postulados teóricos de los niveles de prevención de Leavell \& Clark 4 , el cual se sustenta en el desarrollo de una serie de actividades puntuales de rehabilitación y recuperación específica de un daño de la salud a nivel individual.

La visión de este paradigma se orienta en la planificación normativa, vinculante a la esencia de una educación bancaria, donde la construcción y transferencia de conocimientos es a partir de la hegemonía de un pequeño grupo. Aspecto que se identifica con la orientación de las acciones de educación que se realizan en el marco del programa de control de la leishmaniasis en los servicios, como en las comunidades, donde los equipos de salud a partir de sus conocimientos técnicos y científicos, diseñan los mensajes educativos sin considerar las expectativas y necesidades reales de la población a las cuales van dirigidos. Otras características que identifican este paradigma se relacionan con la definición y aplicabilidad de los conceptos de espacio y tiempo, como factores en la gestión de las acciones de educación para la salud. En este caso, se parte de los escenarios generalistas, sin consideraciones particulares de los espacios específicos de las culturas de los grupos de población, como el desconocimiento de la historia sociocultural y política de las comunidades, el énfasis de la utilización de recursos didácticos, en general, con un contenido generalista y masivo, sin ningún tipo de criterios metodológicos y análisis de contextos socioculturales de los públicos/objetivos particulares a los cuales van dirigidos. Y la ausencia de una matriz de indicadores de gestión que permita evaluar en forma oportuna los resultados e impacto de las acciones de educación para la salud.

Esta visión de educación para la salud manejada cotidianamente desde los programas de control de leishmaniasis en Venezuela, no es más que la reproducción del modelo de educación sanitaria, cuyos resultados han demostrado tener profundas deficiencias para generar un impacto y sostenibilidad en las transformaciones de conducta individual y colectiva referente a la salud. Indicando con ello que es necesario apuntar hacia nuevos paradigmas que guarden consistencia con los cambios y características socio-históricas del país, y así poder construir en equipo espacios sostenibles de educación y participación comunitaria que contribuyan a minimizar el impacto de la resurgencia de las enfermedades infecciosas.

Por lo tanto, el paradigma de educación para la salud propuesto tiene su orientación filosófica en la promoción y calidad de vida de la población, sustentado en un marco de políticas públicas y de salud. Esto lleva obligatoriamente a cambiar el modelo biologicista-unicausal, donde se privilegia la enfermedad, y nos ubica en la dimensión de la salud. Así se puede internalizar y aplicar acciones de educación para la salud desde la comprensión de los diferentes indicadores de desarrollo social que favorecen la presencia de la leishmaniasis en las diferentes comunidades.

En esta línea de pensamiento se integra el modelo de educación para la salud sustentado en el método etnográfico, y complementado por un sistema de georreferencia y un modelo epidemiológico histórico y social (Figura 3).

Figura 3

Modelo de educación para la salud.

Educación para la salud

$\Downarrow$

Etnografía $\Longleftrightarrow$ Sistema de georreferencia $\Longleftrightarrow$ Epidemiología

\section{Consideraciones finales}

Si partimos de que el resurgimiento de las enfermedades infecciosas en América Latina y en Venezuela en forma particular, obedece a la presencia e interrelación de múltiples factores, entre los cuales cobra mucha fuerza las inequidades y las desigualdades sociales, debemos asumir que el desafío hacia el cambio de esa realidad, no solamente es a partir de estrategias de control clínico y epidemiológico. Las mismas son impor- 
tantes, pero deben ser analizadas y reformuladas desde la perspectiva de políticas integrales de promoción, educación y calidad de vida de las poblaciones endémicas.

Esto indica que la inversión de recursos hacia iniciativas de programas de control de endemias en los países de la región, debe evaluarse en cuanto a la orientación de los mismos, y verificar el apoyo real con que se cuenta para desarrollar iniciativas de educación para la salud y participación comunitaria bajo nuevos enfoques metodológicos, donde juega papel importante el sentido de la transdisciplinariedad y transectorialidad.

\section{Resumen}

Este artículo presenta la experiencia de validación de la metodología etnográfica como una alternativa de aproximación objetiva y oportuna en la construcción de escenarios socioculturales de las comunidades afectadas por la leishmaniasis cutánea en Venezuela. Comienza con una breve presentación del resurgimiento de la leishmaniasis en las Américas y en Venezuela en el contexto de las desigualdades sociales. Continúa con una revisión del papel de la educación para la salud en los programas de control de endemias. Luego destaca el papel de la etnografía en la construcción de la racionalidad de la vida cotidiana, sus significados $e$ interpretación de las personas que sufren la enfermedad, para la elaboración de programas de educación para la salud permanente y sostenible. La validación de la metodología se hizo a través de un taller, donde se hizo un análisis de la historia clínica y epidemiológica utilizada habitualmente para recoger información sobre la enfermedad. Entre los principales hallazgos se encuentra la inoperancia del diseño de la historia clínica para la obtención de información relativa a los factores socioculturales vinculantes a la enfermedad, expresada por territorios geográficos específicos con el apoyo de la georreferencia.

Educación en Salud; Participación Comunitaria; Antropología Cultural; Leishmaniasis; Iniquidad Social

\section{Referencias}

1. Organización Panamericana de la Salud. Epidemiología y control de la leishmaniasis en las Américas por país o territorio. Cuaderno 44. http:/ / www. paho.org/spanish/ad/dpc/cd/leishmaniasis.htm (accedido el Jul/2003).

2. Instituto de Biomedicina. Registro nacional de leishmaniasis. Caracas: Instituto de Biomedicina; 2002.

3. Breilh J. Deterioro de la vida: un instrumento para el análisis de prioridades en lo social y la salud. Quito: Editora Nacional; 1990.

4. García JC. Pensamiento social en salud en América Latina. México DF: Interamericana McGraw-Hill/ Organización Panamericana de la Salud; 1994.

5. Freire P. La naturaleza política de la educación. Barcelona: Editorial Paidós; 1990.

6. Granada-Echeverry P. La etnografía en la práctica médica. Revista de Ciencias Humanas 1998; 31: 57-66.

Recibido el 09/Nov/2005

Versión final presentada el 12/Dic/2006

Aprobado el 20/Dic/2006 